\title{
Resolving the Surfaces of Extrasolar Planets with Secondary Eclipse Light Curves
}

\section{Citation}

Williams, Peter K. G., David Charbonneau, Curtis S. Cooper, Adam P. Showman, and Jonathan J. Fortney. 2006. "Resolving the Surfaces of Extrasolar Planets with Secondary Eclipse Light Curves." The Astrophysical Journal 649 (2): 1020-27. https://doi.org/10.1086/506468.

\section{Permanent link}

http://nrs.harvard.edu/urn-3:HUL.InstRepos:41397394

\section{Terms of Use}

This article was downloaded from Harvard University's DASH repository, and is made available under the terms and conditions applicable to Other Posted Material, as set forth at http:// nrs.harvard.edu/urn-3:HUL.InstRepos:dash.current.terms-of-use\#LAA

\section{Share Your Story}

The Harvard community has made this article openly available.

Please share how this access benefits you. Submit a story.

Accessibility 
The Astrophysical Journal, 649:1020-1027, 2006 October 1

(C) 2006. The American Astronomical Society. All rights reserved. Printed in U.S.A.

\title{
RESOLVING THE SURFACES OF EXTRASOLAR PLANETS WITH SECONDARY ECLIPSE LIGHT CURVES
}

Peter K. G. Williams, ${ }^{1}$ David Charbonneau, ${ }^{1}$ Curtis S. Cooper, ${ }^{2}$ Adam P. Showman, ${ }^{2}$ and Jonathan J. Fortney ${ }^{3}$ Received 2006 January 4; accepted 2006 June 22

\begin{abstract}
We present a method that employs the secondary eclipse light curves of transiting extrasolar planets to probe the spatial variation of their thermal emission. This technique permits an observer to resolve the surface of the planet without the need to spatially isolate its light from that of the central star. We evaluate the feasibility of this technique for the HD 209458 system by simulating observations made with the Spitzer Infrared Array Camera (IRAC). We consider two representations of the planetary thermal emission: a simple model parameterized by a sinusoidal dependence on longitude and latitude, and the results of a three-dimensional dynamical simulation of the planetary atmosphere previously published by Cooper \& Showman. We find that observations of the secondary eclipse light curve are most sensitive to a longitudinal asymmetry in the dayside planetary emission. To quantify this signal, we define a new parameter, the "uniform time offset," which measures the time lag between the observed secondary eclipse and that predicted by a planet with spatially uniform emission. We compare the predicted amplitude of this parameter for HD 20948 with the precision with which it could be measured with IRAC. We find that IRAC observations at $3.6 \mu \mathrm{m}$ of a single secondary eclipse should permit sufficient precision to confirm or reject the Cooper \& Showman model of the surface flux distribution for this planet. We quantify the signal-to-noise ratio for this offset in the remaining IRAC bands and find that a modest improvement in photometric precision should permit a similarly robust detection.
\end{abstract}

Subject headings: binaries: eclipsing — infrared: stars — planetary systems — stars: individual (HD 209458) — techniques: high angular resolution — techniques: photometric

\section{INTRODUCTION}

The identification of the first transiting extrasolar planet, HD 209458b (Charbonneau et al. 2000; Henry et al. 2000; Mazeh et al. 2000), initiated a flurry of investigations into the properties of the planetary body that are not possible for nontransiting objects. Nine transiting extrasolar planets have now been identified; for a review of their properties, see Charbonneau et al. (2006). In addition to HD 209458b, three of these (TrES-1, Alonso et al. 2004; HD 149026b, Sato et al. 2005; and HD 189733b, Bouchy et al. 2005) orbit stars that are sufficiently close, and hence bright enough $(V<12)$, to permit a direct study of their atmospheric absorption and emission features through a variety of techniques. One such method is transmission spectroscopy, whereby stellar spectra gathered outside and inside times of planetary transit are compared, in order to search for additional absorption features in the latter due to the presence of certain atoms or molecules in the planetary atmosphere. The only detections of this effect to date have been achieved with the STIS spectrograph aboard the Hubble Space Telescope: Charbonneau et al. (2002) observed HD 209458 in visible light and detected the absorption from gaseous atomic sodium in the planetary atmosphere, and VidalMadjar et al. (2003) observed the same system in the ultraviolet to detect absorption resulting from a large cloud of atomic hydrogen escaping from the planet. Numerous ground-based observational efforts (Bundy \& Marcy 2000; Moutou et al. 2001, 2003; Brown et al. 2002; Winn et al. 2004; Narita et al. 2005) have yielded only upper limits, albeit useful ones. Most recently, Deming et al. (2005a) placed a stringent upper limit on the pres-

\footnotetext{
1 Harvard-Smithsonian Center for Astrophysics, 60 Garden Street, Cambridge, MA 02138; pwilliams@cfa.harvard.edu,dcharbonneau@cfa.harvard.edu.

2 Department of Planetary Sciences and Lunar and Planetary Laboratory, University of Arizona, 1629 University Boulevard, Tucson, AZ 85721; curtis@ 1pl.arizona.edu, showman@1pl.arizona.edu.

3 Space Science and Astrobiology Division, NASA Ames Research Center, MS 245-3, Moffett Field, CA 94035; jfortney@arc.nasa.gov.
}

ence of gaseous CO from observations near $2.3 \mu \mathrm{m}$. Along with the sodium detection, these observations place tight constraints on the distribution of condensates in the upper atmosphere (Fortney 2005).

A complementary technique that promises to be at least as powerful is occultation photometry and spectroscopy. This method subtracts observations gathered during secondary eclipse (i.e., when the planet passes behind the star) from those gathered just before or after this time (when the planet is unocculted), to search for any excess emission attributable to the planet itself. For a hot Jupiter planet orbiting a Sun-like star, the relative size of this excess at infrared wavelengths is a few parts in one thousand. Ground-based attempts to observe thermal radiation from transiting planets have been frustrated by the variability of the telluric opacity over short timescales, and the large ambient thermal background, and thus have resulted in only upper limits (Wiedemann et al. 2001; Lucas \& Roche 2002; Richardson et al. 2003a, 2003b). Recently, two groups have succeeded in detecting the planetary thermal emission; once again this feat was enabled by a space-based observatory, in this case the Spitzer Space Telescope (Werner et al. 2004). Charbonneau et al. (2005) used the Infrared Array Camera (IRAC; Fazio et al. 2004) to detect the thermal emission of TrES-1 in two band passes, $4.5 \mu \mathrm{m}$ and $8.0 \mu \mathrm{m}$. Deming et al. (2005b) used the Multiband Imaging Photometer (MIPS; Reike et al. 2004) to detect the thermal emission from HD 209458b at $24 \mu \mathrm{m}$, and Deming et al. (2006) employed the Infrared Spectrograph (IRS; Houck et al. 2004) to detect the emission from HD $189733 \mathrm{~b}$ at $16 \mu \mathrm{m}$. Each of the data sets consisted of a rapid cadence, high signal-to-noise ratio $(\mathrm{S} / \mathrm{N})$ photometric light curve spanning a predicted time of secondary eclipse, in which a decrement in the total system flux of the expected depth and duration was clearly detected at the anticipated time.

An intriguing possibility permitted by such observations is that of resolving the surface of the planet through high-S / N photometry during the times of ingress and egress, when different 
portions of the planetary surface are occulted by the star. The purpose of this paper is to explore this effect in detail, and to evaluate the likelihood of its detection with the Spitzer Space Telescope.

It is not currently possible to directly image exoplanetary surfaces, nor is this ability anticipated for any planned facility, including the NASA Terrestrial Planet Finder. Nonetheless, the spatial dependence of the planetary photosphere is accessible to an observer through careful monitoring of the structure in a secondary-eclipse light curve. During the ingress and egress phases of secondary eclipse, which last approximately 15-30 minutes, depending on the orbital geometry and planetary radius, the portion of the visible planetary hemisphere (the "dayside") that remains unocculted varies smoothly as a function of time. From the known system parameters (namely the orbital period, phase, inclination, and radii of the planet and star), it is then possible to invert the observed light curve to recover some aspects of the flux distribution across the dayside of the planet. This technique is not new; a similar approach has been used, for example, to produce surface maps of Pluto and Charon (for a review for such observations, see Stern 1992). But only with the Spitzer detections of the past year has it been feasible to consider applying this technique to extrasolar planets.

This possibility is particularly interesting because several recent dynamical simulations have predicted the presence of a large flux contrast across the dayside of a hot Jupiter planet. Independent studies of HD 209458b by Showman \& Guillot (2002) and Cooper \& Showman (2005, hereafter CS05) predict a strong eastward jet. The CS05 supersonic jet pushes the atmosphere's hottest region downstream by $60^{\circ}$ from the planetary substellar point. The planetary circulation pattern leads to global temperature variations of $\sim 500 \mathrm{~K}$ at photospheric pressures. Simulations by Cho et al. (2003) find three broad east-west jets and polar vortex motions. They find that temperature variations may reach up to $\sim 1000 \mathrm{~K}$ in certain circumstances. Burkert et al. (2005) find similarly large temperature variations. They highlight how atmospheric opacity, which controls the penetration depth of stellar flux, affects the circulation problem. Rapid cadence light curves during ingress and egress may allow for observational tests of these circulation models. The analysis of such light curves would provide insight into the dynamical flows of hot Jupiters and also illuminate the energy budgets of these planets.

In $\S 2$, we provide further motivation for the use of a secondary eclipse light curve to study a planetary surface flux distribution. In $\S 3$, we describe the software that we developed for simulating light curves, which forms the basis of our subsequent investigations. In $\S 4$, we introduce a parameter, the "uniform time offset," that characterizes the longitudinal flux contrast of a planetary emission. We then probe the behavior of this parameter with Monte Carlo simulations of hypothetical light curves. Finally, in $\S 5$, we discuss our results and near-future applications of this technique. In this paper, we restrict our focus to upcoming observations of HD 209458b with the Spitzer IRAC instrument. It should be emphasized, however, that our technique can be applied to other instruments and transiting extrasolar planets.

\section{MOTIVATION}

The key concept behind this paper is that the shape of the ingress and egress portions of a secondary eclipse light curve convey information about the spatial variation of the flux emitted across the visible hemisphere of a planet. This is because the disk of the primary gradually and predictably obscures the disk of the planet during secondary eclipse. If the geometry of the orbit is known, at a given point in time the observer knows exactly which region of the planet is obscured. The time variation in the light curve thus becomes a proxy for the spatial variation in emitted flux over the unobscured region of the planetary photosphere.

For an observer, the issue is how one infers a flux distribution from an observed light curve. It is not possible to map uniquely from the latter to the former, as the one-dimensional light curve can convey only an integrated version of the planet's two-dimensional emission. Gross surface features, however, can be inferred from a light curve. For instance, a relatively large and rapid increase in flux during egress indicates that a bright spot on the planet was just unobscured. The location of this spot on the planetary surface can be partially constrained based on the known orbital geometry; for example, neglecting the curvature of the stellar limb across the planetary surface, and approximating the orbital inclination as $90^{\circ}$, the longitude of the spot can be determined, but the latitude will remain unknown. A noteworthy feature of secondary eclipse light curves is that their ingress and egress portions provide complementary information about the spatial variation of planetary flux, since ingress and egress permit distinct regions of the planet to be viewed in isolation. An effective way of extracting information from a noisy eclipse light curve is to compare it to the output of a model; that is, to test whether a light curve is consistent with a given underlying model of the planet surface flux distribution. This requires a well-defined measure of consistency that will be useful in the face of realistic photometric noise that may partially corrupt the features of the ingress and egress portions of a light curve.

An additional concern is that of planetary rotation. In principle, this method could be used to directly determine the rotation period of the hot Jupiter, by observing the change in location on the visible hemisphere of a feature during the elapsed time between ingress and egress (typically $1.5-3 \mathrm{hr}$, depending on the orbital geometry and stellar radius). Hot Jupiters are expected to be tidally locked, however, in which case the planet rotates only slightly during this time (specifically, HD 209458b rotates $13^{\circ}$ during the eclipse). Although we include the effect of such rotation in our calculations below, we note that during ingress and egress a tidally locked planet presents essentially the same face, thus simplifying interpretation of any observed structure in the eclipse light curve.

\section{MODELING LIGHT CURVES}

To simulate the eclipse light curve, we first calculate a series of resolved flux images of the planet as a function of orbital phase. Each flux image is then converted into a single flux value by masking out the portion of the planet that would be obscured by the primary and summing the unmasked pixels; a complete light curve is composed of a sequence of these flux values. The flux values are then normalized to a predefined eclipse depth, to match the expected planet-to-star flux ratio.

In all of our simulations, we fixed the orbital and system parameters to those of the HD 209458 system (§ 3.3). Each light curve is a function of the underlying model of the planetary emission, and the spectral response function of the particular IRAC bandpass ( $(3.2)$, which is required to calculate the relative number of photons generated by each pixel. We consider three models for the spatial dependence of the planetary emission, of increasing complexity: (1) a uniformly emitting disk, (2) a sinusoidal variation in longitude and latitude, and (3) a numerical, dynamical simulation of the atmosphere of HD 209458b. For each of these three emission models, we generated four light curves, one for each bandpass of IRAC. These individual steps are described in greater detail in the following sections. 


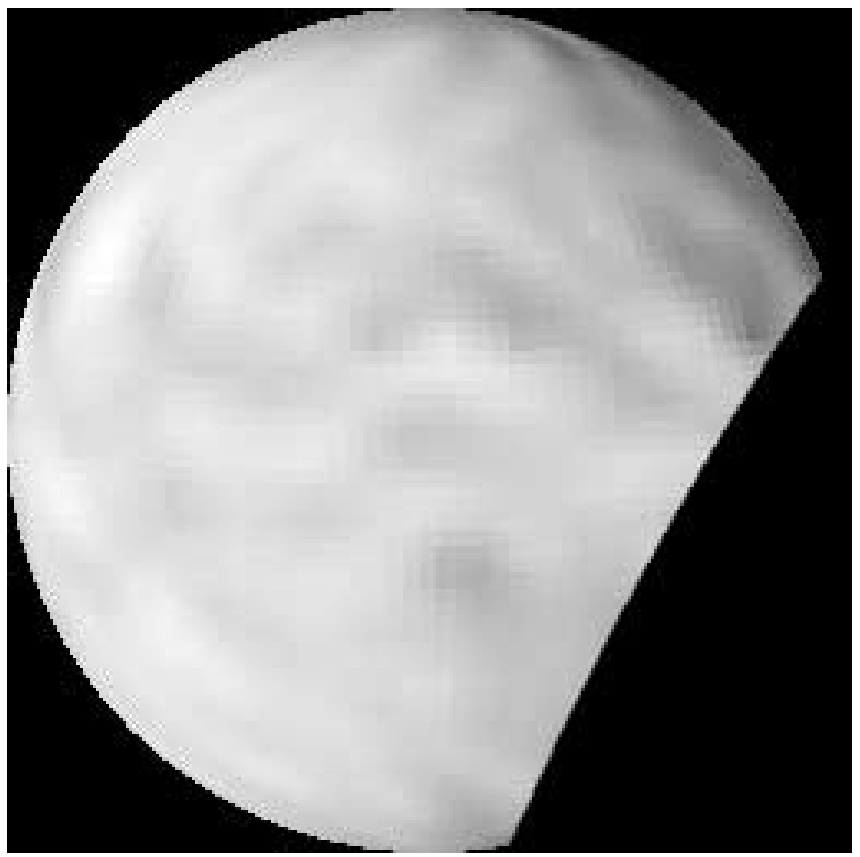

FIG. 1. - Example flux image simulating the appearance of HD $209458 \mathrm{~b}$ as described by the CS05 model in the $8.0 \mu \mathrm{m}$ IRAC bandpass at a time 5 minutes after the start of ingress. The photospheric pressure for this bandpass is 24 mbar.

\subsection{Flux Images}

Each flux image depicts the planet as a disk projected onto a $256 \times 256$ pixel grid; a greater resolution does not alter the resulting light curve, as the underlying models for the spatial dependence of the flux do not vary on finer spatial scales. For the uniform emission model, every pixel in the disk is set to unity. In the other cases, the value of each pixel in the disk is derived from a flux map of the emission of the planet. The coordinates of each pixel are reverse mapped to a latitude $\phi$ and longitude $\lambda$, using an inverse orthographic projection in which the orientation of the planet is determined from the geometry of the orbit and the assumption that the planet is tidally locked with its primary. The point $(\phi=0, \lambda=0)$ is defined to be the substellar point. In the sinusoidal model, the flux map is specified as

$$
F_{A, B}(\phi, \lambda)=A \cos (\phi)+B \sin (\lambda)+1,
$$

where $A$ and $B$ are parameters that specify the latitudinal and longitudinal contrast, respectively.

In the third case, we employ the results of the CS05 simulation, which modeled HD 209458b's atmosphere as a gas in three dimensions, with columns in hydrostatic equilibrium. Their model predicts a superrotating zonal jet dominating the flow at the equator and midlatitudes. This jet blows the hottest regions of the atmosphere downstream from the substellar point by about $60^{\circ}$. At the time of the secondary eclipse, this appears as a large, hot region near the planet's trailing edge. CS05 ran the simulation with a resolution of 45 points in latitude, 72 points in longitude, and 40 vertical layers logarithmically spaced from $1 \mathrm{mbar}$ to $1 \mathrm{kbar}$. The output of their model is the temperature and pressure at each of these locations. We interpolated the output of the CS05 model onto a grid in longitude and latitude of twice their original resolution, and then to each of our $256 \times 256$ points we associated the nearest interpolated CS05 grid point, and assigned a flux value using the method described in $\S 3.2$. Figure 1 shows an example flux image derived from the CS05 model.
We also considered the simulation of HD 209458b developed by Cho et al. (2003), who modeled the planetary atmosphere as a frictionless two-dimensional gas in hydrostatic balance (in contrast to the three-dimensional model of CS05). Unlike the results for $\mathrm{CS} 05$ model, the Cho et al. model resulted in only very weak perturbations to the secondary eclipse light curve. Since such a light curve could not be distinguished from a uniformly emitting disk (given foreseeable observational errors), we did not pursue this model further.

\subsection{Temperature to Flux Conversion for the CS05 Model}

In order to generate flux images, the pressure-temperature $(p-T)$ profiles of the CS05 model must to be converted to flux values. Formally, this would require us to solve the equation of radiative transfer in a dynamical, spatially varying model of the chemistry of HD 209458b's atmosphere. Since our goal in this paper is to present a first estimate of the perturbations to the secondary eclipse light curve, we simplified the problem using the approach described below. We model the emission of each atmospheric column as a blackbody emitting isotropically at the temperature in the $p-T$ profile corresponding to the photospheric pressure. Photospheric pressures for various wavelengths were previously determined from radiative transfer calculations by Fortney et al. (2005). Using the same one-dimensional HD 209458 b model, we computed the brightness temperature in each of the IRAC band passes, taking into account each band's transmission function. ${ }^{4}$ The brightness temperatures are different in each of the four bands and are sensitive to atmospheric opacity. We then compared these brightness temperatures to the Fortney et al. $p$ - $T$ profile to obtain the atmospheric pressure that corresponds to each temperature. One can think of each of these pressures as the "photospheric pressure" in each band. Assuming solar composition and approximate radiative equilibrium, we find resulting photospheric pressures of $95 \mathrm{mbar}(3.6 \mu \mathrm{m}), 48 \mathrm{mbar}$ $(4.5 \mu \mathrm{m}), 32 \mathrm{mbar}(5.8 \mu \mathrm{m})$, and $27 \mathrm{mbar}(8.0 \mu \mathrm{m})$. Four brightness temperature maps were then generated by taking the temperatures from the pressure levels in the CS05 tables nearest these photospheric pressures: 105, 50, 35, and 24 mbar, respectively. These maps were converted to final flux maps by integrating the IRAC response functions over blackbody emission at those temperatures.

This approach is approximate, because the photospheric pressures were calculated using the Fortney et al. (2005) radiativeequilibrium $p-T$ profiles rather than the weather-modified $p-T$ profiles from CS05. Nevertheless, the temperature dependences of the opacities are modest, so the photospheric pressures calculated in this manner should provide reasonable approximations for the CS05 $p$ - $T$ profiles. A significant uncertainty is the existence of clouds: an opaque cloud at a few mbar pressure, for example, would move the photosphere to the cloud-top pressure in all four bands. We have assumed that such clouds are absent. We note that significant opacity variations on isobars are possible in principle, due to lateral variations in the concentrations of $\mathrm{CO}$ and $\mathrm{CH}_{4}$ in the upper layers of the planetary atmosphere. Recent simulations by Cooper \& Showman (2006), however, show that the concentrations of $\mathrm{CO}$ and $\mathrm{CH}_{4}$ are likely homogenized above the 1 bar level. This lends credence to our adoption of a single pressure level identifying the photosphere in each bandpass. We also note that our emission model ignores effects due to the slant path for emission from the limb of the planet, i.e., limb darkening. Fortney et al. (2006a) perform detailed radiative transfer calculations for the Cooper \& Showman (2006) dynamical atmosphere

\footnotetext{
4 See http://ssc.spitzer.caltech.edu/irac/spectral_response.html.
} 

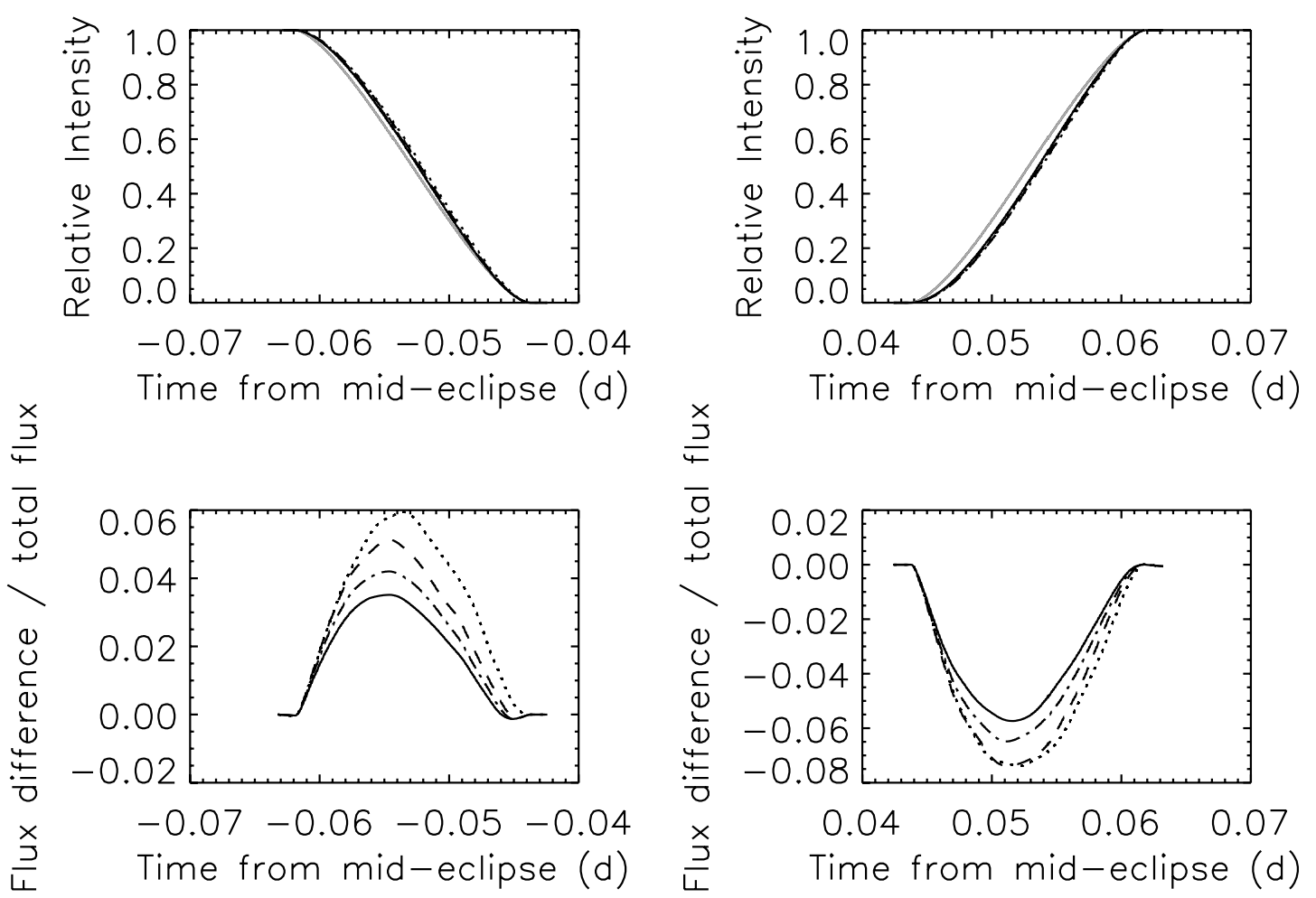

FIG. 2.-Upper left: Ingress portion of the secondary eclipse light curves of HD 209458b, assuming a uniform flux distribution (solid gray line) and the CS05 model for the spatial flux variation (dashed lines overlying each other). Lower left: The relative differences in the curves resulting from the CS05 model to the prediction of the uniform flux distribution, for the four IRAC band passes ( $3.6 \mu \mathrm{m}$, dotted lines; $4.5 \mu \mathrm{m}$, dashed lines; $5.8 \mu \mathrm{m}$, dash-dotted lines; and $8.0 \mu \mathrm{m}$, solid lines). The right panels depict these curves and their differences at egress.

model of HD 209458b. Using the same methods, we have analyzed the CS05 grid and find an effect equivalent to a temperature change of $-100 \mathrm{~K}$ at the planetary limb. We attribute this small degree of limb darkening to the fact that the dayside atmospheric profiles of the CS05 and Cooper \& Showman (2006) grids are nearly isothermal; isothermal atmospheres show no limb darkening. We discuss in $\S 5$ why this small effect can safely be ignored.

\subsection{Light Curve Generation}

In each flux image, the portion of the planet obscured by the primary is masked out to yield an image similar to Figure 1. The position of the star relative to the planet is calculated using the published system parameters: period of 3.52474 days, inclination $i=$ 86.6, primary mass $M_{*}=1.1 M_{\odot}$, stellar radius $R_{*}=1.12 R_{\odot}$, and planetary radius $R_{P}=1.32 R_{J}$. We assumed a circular orbit, consistent with the most recent radial-velocity data (Laughlin et al. 2005) and the timing of the secondary eclipse (Deming et al. 2005b). We represented the star as a geometric circle with its center specified by the projected separation between the star and planet. Each pixel in the flux image was scaled by $n / 4$, where $n$ was the number of the pixel's corners not inside the circle. The resulting light curve was then renormalized to account for the flux ratio of the star to the planet. The band-dependent depth of the eclipse was taken from theoretical calculations. Fortney et al. (2005) calculated the spectrum for HD 209458b from a radiativeequilibrium atmosphere model, and subsequently estimated the planet-to-star flux ratios in each IRAC bandpass. They predict secondary eclipse depths of $0.00096(3.6 \mu \mathrm{m}), 0.00112(4.5 \mu \mathrm{m})$, $0.00145(5.8 \mu \mathrm{m})$, and $0.00190(8.0 \mu \mathrm{m})$, and we assume these values here. Our resulting light curves for HD $209458 \mathrm{~b}$ are shown in Figure 2.
We note here a few additional concerns. First, we have neglected variations in the stellar flux over the $\sim 6 \mathrm{hr}$ timescales, an assumption which is justified by solar observations (see Batalha et al. 2002; Borucki et al. 2004). We also neglect gravitational lensing by the primary, as calculations by Agol (2002) indicate that this effect is negligible. Finally, the light curves are sensitive to the chemistry and dynamics of the planetary atmosphere. The characteristics of both these aspects of the atmosphere and the interplay between them (e.g., clouds) remain uncertain, but will be further constrained by both future models that self-consistently couple dynamics and radiation and additional Spitzer observations.

\section{EVALUATING THE DETECTABILITY OF SURFACE NONUNIFORMITIES}

The effect of a nonuniform flux distribution on the secondary eclipse light curves (Fig. 2) is subtle. The detailed structure of ingress and egress is likely beyond the reach of current instrumentation. Nonetheless, it may be possible to confirm or exclude the presence of largescale nonuniformities, such as those predicted by the CS05 model. To this end, we define ( $\S 4.1$ ) a robust observational parameter that is sensitive to such non-uniformities, and subsequently, using Monte Carlo simulations (§ 4.2), we evaluate its sensitivity for simulated Spitzer IRAC data ( $\S \S 4.3$ and 4.4).

\subsection{The Uniform Time Offset}

We define the "uniform time offset" $t_{\text {offs }}$ as the time lag that must be applied to a synthetic light curve (generated under the assumption of a uniform planetary flux distribution) to minimize its $\chi^{2}$ difference from the data. This parameter can be interpreted as the longitudinal separation between the photometric 
and geometric centroids of the planet, i.e., the greater this separation, the greater the time lag between the center of the observed light curve (which is a function of the photometric centroid) and that of the predicted light curve (which is a function of the planet's projected location and hence geometric centroid). To first order, a planet's photometric centroid will be off-center if its emission is longitudinally asymmetric, so the uniform time offset is a simple observational gauge of this longitudinal asymmetry. In detail, the time lag will also depend on the orbital inclination and the curvature of the stellar limb across the face of the planet. We explore the behavior of this parameter below.

To estimate the uniform time offset for a given observed time series, we must first generate a synthetic light curve. This uniform light curve $\Phi(t)$ is calculated under the assumption that the planet has a uniform surface flux distribution, and hence its tabulation requires only a knowledge of the orbital parameters and the radii of the planet and star. The eclipse depth is then normalized to match the estimated depth of the observed time series. Synthetic data points only need to be generated for the ingress and egress portions of the transit, since the assumed uniformflux model reveals no structure outside of these times.

In our implementation, we generated $\Phi(t)$ on a grid of times from $30 \mathrm{~s}$ prior to ingress to $30 \mathrm{~s}$ after egress; this was smoothed with a boxcar function to eliminate any discrete pixel effects resulting from our adoption of a $256 \times 256$ grid, and subsequently interpolated to a spacing of $0.1 \mathrm{~s}$. Once the synthetic light curve has been generated, the uniform time offset can be evaluated by minimizing the $\chi^{2}$ value of the model $\Phi(t)$ to the observed time series

$$
\chi^{2}(\tau)=\frac{1}{\sigma_{\mathrm{obs}}^{2}} \sum_{i}\left[F_{i}-\Phi\left(t_{i}+\tau\right)\right]^{2},
$$

where $\tau$ is the time shift being applied, $F_{i}$ is the observed relative flux value of the $i$ th data point, and $t_{i}$ is the time associated with that point. In our implementation, every point in the observed light curve is given equal weight. We note that a possible variation would be to determine distinct uniform time offsets for the ingress and egress portions of a light curve, which would allow the observer to investigate any differences between these two events.

To illustrate how the uniform-time offset encodes information about the flux distribution, consider the synthetic, noiseless curves shown in Figure 2. The uniform time offsets for each of these are $-86 \mathrm{~s}(3.6 \mu \mathrm{m}),-77 \mathrm{~s}(4.5 \mu \mathrm{m}),-67 \mathrm{~s}(5.8 \mu \mathrm{m})$, and $-57 \mathrm{~s}(8.0 \mu \mathrm{m})$. The negative values imply that the photometric centroid of the planet lags the geometric centroid (and hence the expectations of a uniform flux planet). Specifically, the planet exhibits an IR-bright region on the trailing limb, which is occulted in the later half of the ingress and unocculted in the later half of the egress.

The variation in the amplitude of the timing offset with bandpass encodes critical information about the advection of heat and the strength of winds as a function of depth in the atmosphere. In the CS05 model, the uppermost layers of the atmosphere have a radiative equilibrium time constant that is much shorter than the timescale over which winds act to advect heat across a hemisphere; as a result, the hottest point is coincident with the subsolar point. At greater atmospheric pressure (i.e., deeper in the atmosphere), this timescale increases, and the hottest region is shifted significantly downwind of (lags) the subsolar point. Since decreasing wavelength corresponds to increasing photospheric depth ( $\S 3.2$ ), the bluemost IRAC bands are predicted to show the greatest value for the uniform time offset.

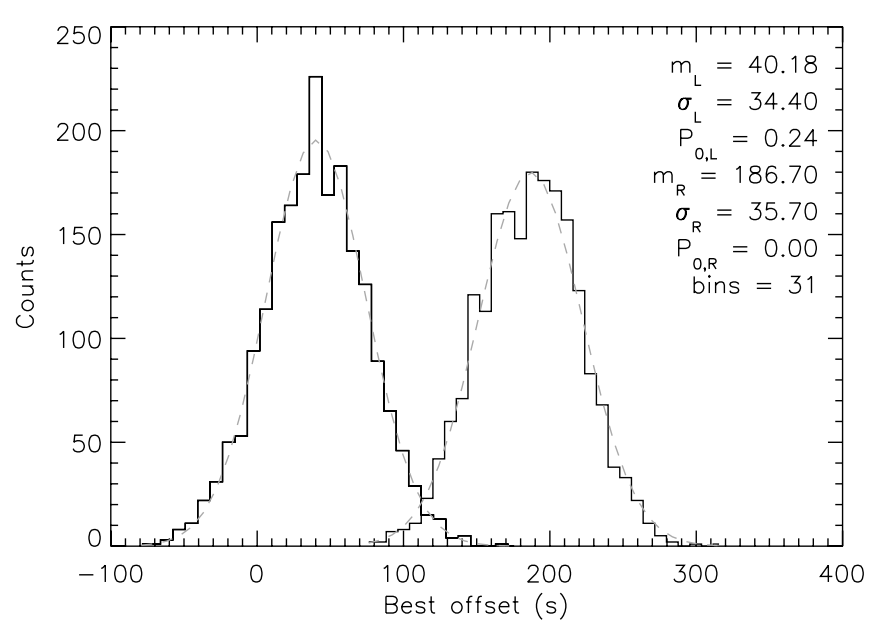

FIG. 3.- Histogram of 2000 best-fit values of the uniform time offset $t_{\text {offs }}$ for two pairs of parameters describing the sinusoidal model, $(A, B)=(0.20,0.20)$ (left solid line) and $(A, B)=(0.10,0.80)$ (right solid line). The observations are simulated in the $4.5 \mu \mathrm{m}$ IRAC band. The dashed curves are Gaussian fits to the histograms, from which the mean $m_{X}$ and error $\sigma_{X}$ were derived, with $X=L$ for the left curve and $X=R$ for the right curve. The probability value $P_{0, L}$ for the left curve is 0.24 , and hence an observed value of $t_{\text {offs }}=0$ cannot rule out this model. For the right curve, $P_{0, R}$ is essentially zero, and thus an observed value of $t_{\text {offs }}=0$ would exclude this model with a high degree of confidence.

\subsection{Monte Carlo Simulations with Synthetic Observations}

We generated synthetic IRAC observations of HD 209458b to explore the detectability of the uniform time offset parameter. We chose this facility because it is the optimal observatory for such observations, and we can ground our calculations in the practical experience of Charbonneau et al. (2005). We created synthetic light curves spanning 0.1 days from the center of eclipse, with a cadence of $15 \mathrm{~s}$. To each model value, we added Gaussian noise as estimated from the Spitzer Observer's Manual. ${ }^{5}$ These predictions are dominated by the photon noise from the star, with a relative noise amplitude $\sigma_{\text {obs }}$ of $0.000323(3.6 \mu \mathrm{m}), 0.000422(4.5 \mu \mathrm{m})$, $0.001041(5.8 \mu \mathrm{m})$, and $0.000831(8.0 \mu \mathrm{m})$. We subsequently estimated the best-fit value of the uniform time offset $t_{\text {offs }}$ by minimizing equation (2). We then conduct a Monte Carlo simulation by repeating this procedure several thousand times, and creating a histogram of the derived values of $t_{\text {offs }}$. The resulting histogram is well approximated by a Gaussian, because the uniform time offsets derive from a simple fit to a large number of data points, the noise properties of which are described by a Gaussian distribution.

The resulting histogram then permits us to directly evaluate the likelihood of detecting a non-zero value for $t_{\text {offs }}$ for a given underlying model for the surface flux distribution. We fit a Gaussian to the histogram to estimate its mean $m$ and standard deviation $\sigma$. If a uniform time offset of $t_{\text {offs }}$ is calculated for some set of observations, the probability $P$ of having obtained such a value if the underlying model is correct decreases as $\left|m-t_{\text {offs }}\right| / \sigma$. We define $P$ to be the probability of making an observation at least as far away from the expected mean as the observed $t_{\mathrm{offs}}$, which is given by the complimentary error function $\operatorname{erfc}\left(\left|m-t_{\text {offs }}\right| / \sqrt{2} \sigma\right)$. We then consider this quantity for $t_{\mathrm{offs}}=0$ (which we denote $P_{0}$ ) associated with a given model and set of parameters; $P_{0}$ is the likelihood that we would observe a $t_{\text {offs }}$ of zero if the underlying model is correct. If $P_{0}$ is small enough, then an observed $t_{\text {offs }}$ of zero would permit us to rule out the model under consideration. Conversely, if we find that $P_{0}$ is not sufficiently small, then an observed $t_{\text {offs }}$ of zero cannot rule out the model.

\footnotetext{
5 See http://ssc.spitzer.caltech.edu/documents/som.
} 

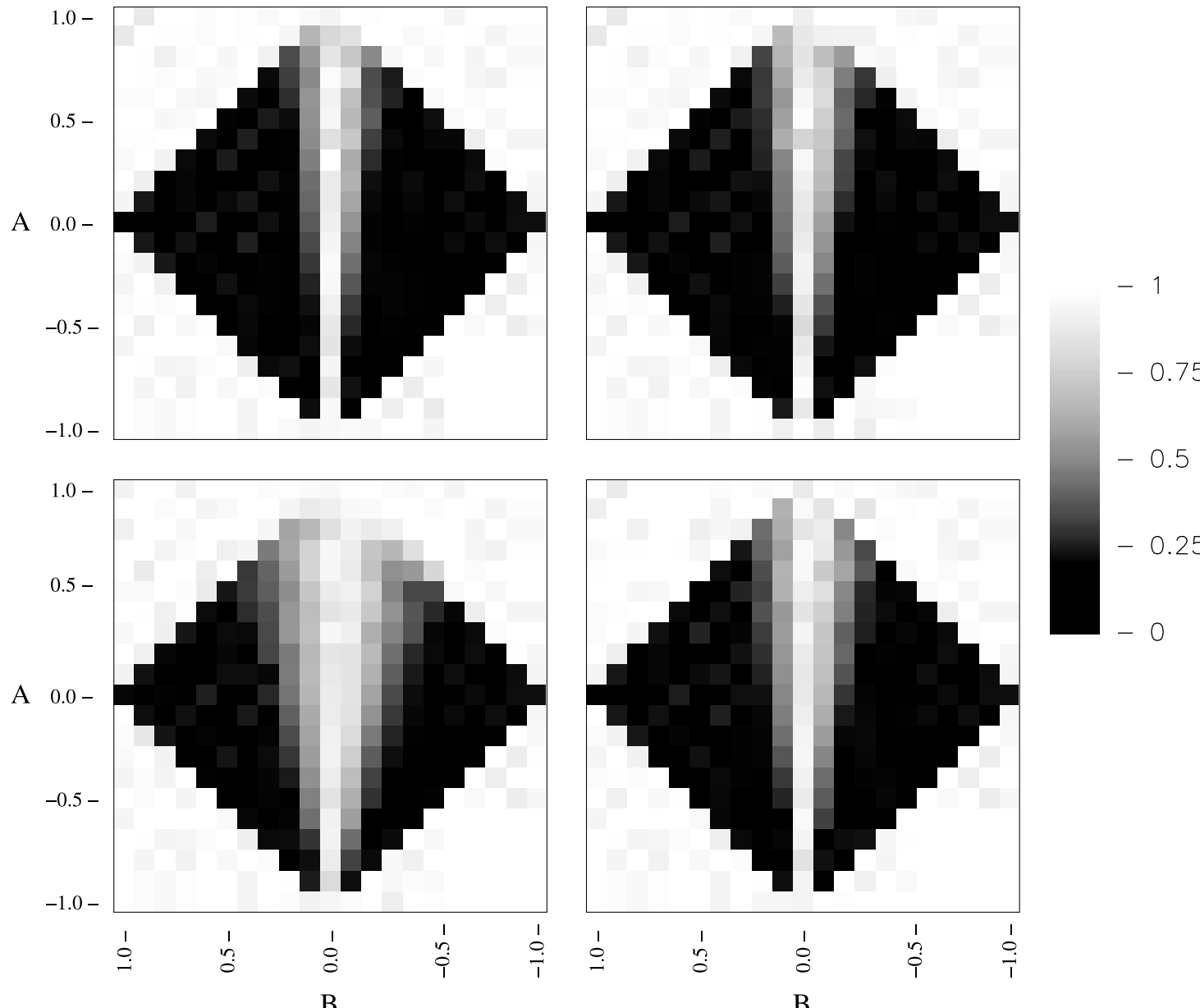

$-0.5$

$-0.25$

B

FIG. 4.-Diamonds representing $P_{0}$ values (§ 4.2) over the $(A, B)$ parameter space of the sinusoidal planetary model as determined by Monte Carlo simulations of IRAC observations (upper left, $3.6 \mu \mathrm{m}$; upper right, $4.5 \mu \mathrm{m}$; lower left, $5.8 \mu \mathrm{m}$; and lower right, $8.0 \mu \mathrm{m}$ ). If a value of $t_{\text {offs }}=0$ were to be observed for the $\mathrm{HD} 209458$ system, models corresponding to the dark squares could be excluded with high confidence, whereas models corresponding to the lighter squares could not. The $A$ parameter controls the latitudinal flux contrast while the $B$ parameter controls the longitudinal flux contrast. Although the dominant sensitivity is to longitudinal contrast, note the effect of the non-equatorial orbit, which permits a modest sensitivity to latitudinal flux variations. Models with $|A|+|B|>1$ were not considered because they would yield areas of the planet presenting a negative flux.

An observed $t_{\text {offs }}$ that is large compared to $\sigma$, however, would be inconsistent with the model.

\subsection{Sinusoidal Model Simulations}

We ran a set of simulations with data generated from the sinusoidal model described in $\S 3.1$. We systematically varied the $A$ and $B$ parameters (and hence the degree of latitudinal and longitudinal contrast) to find the subset of models that could reasonably be detected with Spitzer IRAC observations of HD 209458. We varied $A$ and $B$ from -1 to 1 with increments of 0.1 , considering all pairs for which $|A|+|B|<1$ (values outside this range would result in negative flux values at various points on the planet).

For each $(A, B)$ pair, 2000 simulated data sets were generated, and from each we derived the value of $t_{\text {offs }}$. Figure 3 shows histograms for two examples, $(A, B)=(0.2,0.2)$ and $(0.1,0.8)$, both modeled for the $4.5 \mu \mathrm{m}$ band. The former model is not easily distinguished from one resulting from a uniformly emitting planet, but the latter model has a strong longitudinal contrast that should be readily detectable. Figure 4 summarizes the detectability of given pairs of $(A, B)$ for the four IRAC bandpasses. Indeed, if the planet presents a large longitudinal contrast, we are very unlikely to estimate a zero uniform time offset, while those with low contrast are expected to yield offsets indistinguishable from zero.
The latitudinal contrast has only a weak effect, but interestingly it is not zero; this results from the fact that the orbital inclination of HD $209458 \mathrm{~b}$ is not $90^{\circ}$. As a result, the projected limb of the star across the planet is not symmetric in latitude, and hence the ingress and egress curves encode some information of the latitudinal flux distribution.

\subsection{Observability of CSO5 Model}

We also ran a set of simulations to evaluate whether the CS05 planetary model discussed in $\S 3.1$ could be tested with foreseeable Spitzer IRAC observations. In these simulations, we varied the photometric precision $\sigma_{\text {obs }}$ and evaluated the resulting values for $P_{0}$. These results then permit us to understand to what degree we must improve the photometric precision of such observations to test the CS05 model. We initially used values of $\sigma_{\text {obs }}$, as currently estimated for planned IRAC observations of HD 209458 (as described in $\S 4.2$ ), and then decreased the assumed noise by factors of $4^{1 / 12}$. (We conducted 12 runs, with a net improvement of a factor of 4 in the overall precision.) For each IRAC band and value of $\sigma_{\text {obs }}$, a histogram of 2500 uniform time offsets was generated, from which we calculated the value of $P_{0}$. In Figure 5, we show how $P_{0}$ decreases as a function of the assumed value for $\sigma_{\text {obs }}$ (relative to the nominal value we expect IRAC to deliver) for each of the four IRAC bands. In Table 1, 
TABLE 1

Parameters for the Histogram of Uniform Time Offsets of the CS05 Model

\begin{tabular}{|c|c|c|c|c|}
\hline Parameter & $3.6 \mu \mathrm{m}$ & $4.5 \mu \mathrm{m}$ & $5.8 \mu \mathrm{m}$ & $8.0 \mu \mathrm{m}$ \\
\hline Observational precision, $\sigma_{\mathrm{obs}} \ldots \ldots \ldots \ldots$ & 0.000323 & 0.000422 & 0.001041 & 0.000831 \\
\hline 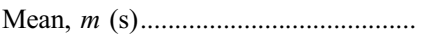 & -91.44 & -80.01 & -76.91 & -62.61 \\
\hline Standard deviation, $\sigma(\mathrm{s}) \ldots \ldots \ldots \ldots \ldots \ldots$ & 34.63 & 38.35 & 72.34 & 43.79 \\
\hline
\end{tabular}

we list the values of $m$ and $\sigma$ describing the resulting histogram of values of $t_{\text {offs }}$ derived for the CSO5 model with the nominal IRAC errors.

We found that an observation of a zero uniform time offset in the $3.6 \mu \mathrm{m}$ IRAC bandpass would already put the CS05 model in doubt, since this would be expected to occur by chance in less than $1 \%$ of such data sets. In the other IRAC bands, errors would need to be improved by factors of roughly $1.3(4.5 \mu \mathrm{m}), 3(5.8 \mu \mathrm{m})$, and $2(8.0 \mu \mathrm{m})$ to achieve a similar level of confidence. Combining the statistical significance of all four bands would permit the observer to confidently exclude the CS05 model should a value of $t_{\text {offs }}$ of zero be observed. We conclude that the uniform time offset technique is a useful tool for testing the predictions of models similar to $\mathrm{CS} 05$, owing to the prominent longitudinal flux contrast that they present.

\section{DISCUSSION AND CONCLUSIONS}

In this paper, we have explored how the secondary eclipse light curves of an extrasolar planet may be used to learn about the spatial variation of its emission. This technique permits an observer to gain access to this important information without the need to directly image to surface of the planet. We have explored this technique with simulations of Spitzer IRAC observations of the planet HD 209458b and considered the resulting value of the uniform time offset parameter $t_{\text {offs }}$ that would be observed if the emission of the planet is consistent with a family of simple models whereby the flux varies sinusoidally in longitude and latitude, as well as the results of a three-dimensional dynamical calculation of the atmosphere of this planet as previously published by CS05.

We found that physically reasonable longitudinal flux contrasts could plausibly be detected in the HD 209458 system with

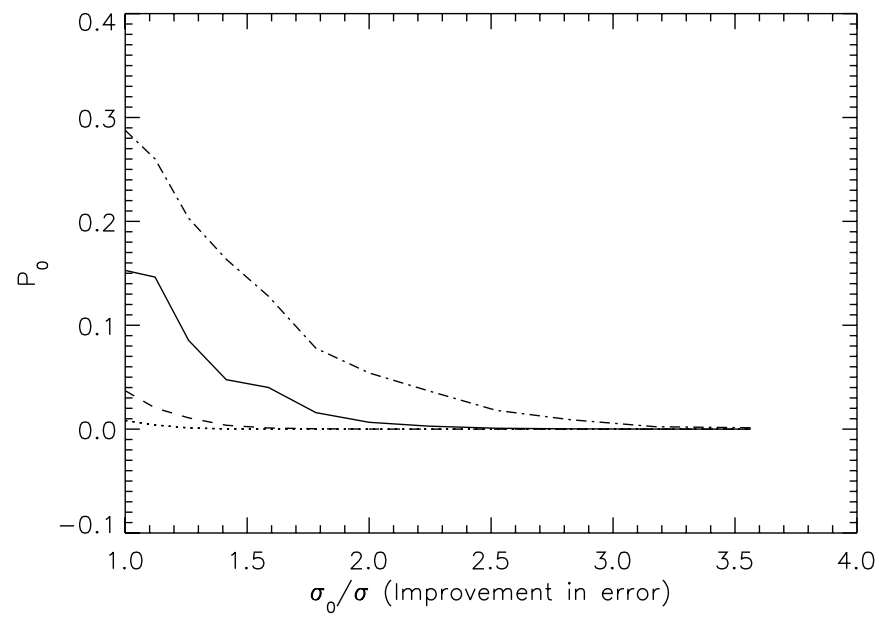

Fig. 5.-Probabilities $P_{0}$ of observing a uniform time offset of 0 if $\mathrm{HD}$ $209458 \mathrm{~b}$ is represented by the CS05 model, as a function of the size of the observational errors relative to their nominal Spitzer IRAC values, $\sigma_{0}$. The four curves correspond to the different IRAC band passes: $3.6 \mu \mathrm{m}$, dotted line; $4.5 \mu \mathrm{m}$, dashed line; $5.8 \mu \mathrm{m}$, dash-dotted line; and $8.0 \mu \mathrm{m}$, solid line. We find that IRAC observations at $3.6 \mu \mathrm{m}$ should provide a robust evaluation of the predictions of the CS05 model. planned Spitzer IRAC observations. Specifically, sinusoidal models with a latitudinal parameter $|B|>0.5$ yield a value of $P_{0}<0.25$ in all but the $5.6 \mu \mathrm{m}$ bands. From the definition of the sinusoidal model, we see that $B=0.5$ corresponds to a flux contrast of a factor of 3 between the leading and trailing edges of the planet. In the IRAC $4.5 \mu \mathrm{m}$ band this contrast would correspond, for example, to two regions emitting as blackbodies with temperatures of approximately 1300 and $900 \mathrm{~K}$. We find that the results of the CS05 model are readily testable with Spitzer IRAC observations. In particular, observations in the $3.6 \mu \mathrm{m}$ band would already be able to exclude this model with a high degree of confidence, should a value of $t_{\mathrm{offs}}=0$ be observed. Of course, interpretation of such data will undoubtedly be more complex than the approach presented here. For example, the presence of high-altitude clouds could decrease the atmospheric pressure corresponding to the photosphere, which would serve to mask the underlying dynamics of the atmosphere (Showman \& Cooper 2006), where the effect of winds on redistributing the energy of the incident stellar flux is much more prominent.

It should be noted that the value of the orbital eccentricity $e$ and longitude of periastron $\omega$ also serve to affect the time of secondary eclipse, which could mimic the effect discussed here. Tidal circularization is expected to reduce the eccentricity of hot Jupiter orbits to virtually zero, in which case observations of the primary eclipse (e.g., Brown et al. 2001) of such systems can generally constrain the predicted time of secondary eclipse to several seconds, which contributes a negligible source of error to the value of $t_{\text {offs }}$. Direct observational constraints on the orbital eccentricity at the required level of precision are likely not feasible, however. The current upper limit on the orbital eccentricity of HD 209458 from the radial velocity observations alone is $e<0.02$ (Laughlin et al. 2005), which could induce an offset in the time of secondary eclipse as large as 65 minutes, more than 2 orders of magnitude greater than the effect we describe. Rather, the validity of the assumption of a circularized orbit can be bolstered by checking for the presence of transit timing variations (Agol et al. 2005; Holman \& Murray 2005), using a sequence of transit observations. The observed lack of such variations (Knutson et al. 2006) indicate the absence of perturbers in the HD 209458 system, thus permitting tidal effects to complete the circularization of the orbit. We also note that the timing offset due to a nonzero orbital eccentricity is fundamentally achromatic, in contrast to the signal we describe here (e.g., Table 1). By observing a single secondary eclipse simultaneously in multiple bandpasses (such as the four bands of the IRAC instrument), an observer can search for variations in the observed value of $t_{\text {offs }}$ between the various wavelength bands, a sure sign that the signal is not due to residual orbital eccentricity. In particular, comparison between the values of $t_{\text {offs }}$ between the $3.6 \mu \mathrm{m}$ and $8.0 \mu \mathrm{m}$ bands would show the largest effect.

There are several ways in which the predictions presented here can be refined. More precise values for the orbital parameters and an observational determination of the eclipse depths could refine our theoretical predictions. But the greatest source of uncertainty in our results is the model of the planetary atmosphere and its 
emission. As mentioned before, clouds might dramatically affect the simulation results. As alluded to in $\S 3.2$, limb darkening is another effect that we do not take into account. Planets other than HD 209458b may even exhibit limb brightening; this possibility has been investigated with regards to the planet HD 149026b by Fortney et al. (2006b). Depending on their assumed model parameters, they produce physically plausible atmospheres that exhibit either limb brightening or limb darkening. In either case, however, the phenomenon is radially symmetric and does not significantly alter the longitudinal flux contrast of the planet, so the effect on $t_{\text {offs }}$ should be modest. We have confirmed this intuition by performing Monte Carlo simulations with the CS05 model modified to have its emission scaled according to the classical limb-darkening law $I^{\prime}(r)=I(r) *\left[1-c\left(1-\sqrt{1-r^{2}}\right)\right]$, where $r \in[0,1]$ is the normalized projected radial distance of a point from the center of the planetary disk and $c$ sets the magnitude of the effect. With $c=0.25$, equivalent to a temperature decrement of $\sim 300 \mathrm{~K}$ on the limb of the planet (which is much larger than what is calculated for HD 209458b), the resulting change in $t_{\text {offs }}$ for all IRAC bands is $\approx 3 \mathrm{~s}$.

In the near future, there will be many opportunities to employ this technique for probing exoplanets. This paper has considered the specific case of observations of HD 209458b with the Spitzer Space Telescope's IRAC. Such observations should yield unprecedentedly precise secondary eclipse light curves, and we conclude that they can realistically be expected to probe the surface flux distribution of the day side of the planet. They would also determine the secondary eclipse depths of HD 209458b in the 4 IRAC bands, which would be of immediate interest in their own right. Even more promising would be observations of the recently discovered extrasolar planet HD 189733b (Bouchy et al. 2005), which is a mere 19 pc away and has a very favorable planet-to-star flux ratio, owing to the relatively large planet-tostar surface area ratio and the large equilibrium temperature of the planet. Spitzer has already observed HD 189733 with all three instruments, and we encourage a search for the effects described in this paper. In the longer term, the James Webb Space Telescope (JWST), with infrared detectors and a larger aperture than Spitzer, will be an extremely powerful tool for applying this technique. As instrumentation and models improve, and as an increasing number of nearby transiting extrasolar planet systems are discovered, the prospects for resolving the surfaces of these distant worlds grows ever brighter.

We would like to thank J. Y.-K. Cho, K. Menou, B. M. S. Hansen, and S. Seager for providing the results from their simulations for use in light curve simulations. We thank the anonymous referee for comments that improved the manuscript. This work is based in part on observations made with the Spitzer Space Telescope, which is operated by the Jet Propulsion Laboratory, at the California Institute of Technology under a contract with NASA. Support for this work was provided by NASA through an award issued by JPL/Caltech. The contributions of C. Cooper and A. Showman were supported by NSF grant AST-0307664 and NASA GSRP NGT5-50462. J. Fortney is supported by a National Research Council Fellowship.
Agol, E. 2002, ApJ, 579, 430

Agol, E., Steffen, J., Sari, R., \& Clarkson, W. 2005, MNRAS, 359, 567

Alonso, R., et al. 2004, ApJ, 613, L153

Batalha, N. M., Jenkins, J., Basri, G. S., Borucki, W. J., \& Koch, D. G. 2002, in Proc. First Eddington Workshop on Stellar Structure and Habitable Planet Finding (ESA SP-485; Noordwijk: ESA), 35

Borucki, W., et al. 2004, in Proc. Second Eddington Workshop on Stellar Structure and Habitable Planet Finding (ESA SP-538; Noordwijk: ESA), 177 Bouchy, F., et al. 2005, A\&A, 444, L15

Brown, T. M., Charbonneau, D., Gilliland, R. L., Noyes, R. W., \& Burrows, A. 2001, ApJ, 552, 699

Brown, T. M., Libbrecht, K. G., \& Charbonneau, D. 2002, PASP, 114, 826

Bundy, K. A., \& Marcy, G. W. 2000, PASP, 112, 1421

Burkert, A., Lin, D. N. C., Bodenheimer, P. H., Jones, C. A., \& Yorke, H. W. 2005, ApJ, 618, 512

Charbonneau, D., Brown, T. M., Burrows, A., \& Laughlin, G. 2006, in Protostars and Planets V, ed. D. Jewitt, K. Keil, \& B. Reipurth, in press (astro-ph/ 0603376)

Charbonneau, D., Brown, T. M., Latham, D. W., \& Mayor, M. 2000, ApJ, 529, L45

Charbonneau, D., Brown, T. M., Noyes, R. W., \& Gilliland, R. L. 2002, ApJ, 568,377

Charbonneau, D., et al. 2005, ApJ, 626, 523

Cho, J. Y.-K., Menou, K., Hansen, B. M. S., \& Seager, S. 2003, ApJ, 587, L117

Cooper, C. S., \& Showman, A. P. 2005, ApJ, 629, L45

2006, ApJ, 649, 1048

Deming, D., Brown, T. M., Charbonneau, D., Harrington, J., \& Richardson, L. J. 2005a, ApJ, 622, 1149

Deming, D., Harrington, J., Seager, S., \& Richardson, L. J. 2006, ApJ, 644, 560

Deming, D., Seager, S., Richardson, L. J., \& Harrington, J. 2005b, Nature, 434, 740

Fazio, G. G., et al. 2004, ApJS, 154, 10

Fortney, J. J. 2005, MNRAS, 364, 649

Fortney, J. J., Cooper, C. S., Showman, A. P., Marley, M. S., \& Freedman, R. S. 2006a, ApJ, in press (astro-ph/0608235)
Fortney, J. J., Marley, M. S., Lodders, K., Saumon, D., \& Freedman, R. S. 2005, ApJ, 627, L69

Fortney, J. J., Saumon, D., Marley, M. S., Lodders, K., \& Freedman, R. S. 2006b, ApJ, 642, 495

Henry, G. W., Marcy, G. W., Butler, R. P., \& Vogt, S. S. 2000, ApJ, 529, L41

Holman, M. J., \& Murray, N. W. 2005, Science, 307, 1288

Houck, J., et al. 2004, ApJS, 154, 18

Knutson, H., Charbonneau, D., Noyes, R. W., Brown, T. M., \& Gilliland, R. L. 2006, ApJ, submitted (astro-ph/0603542)

Laughlin, G., Marcy, G. W., Vogt, S. S., Fischer, D. A., \& Butler, R. P. 2005, ApJ, 629, L121

Lucas, P. W., \& Roche, P. F. 2002, MNRAS, 336, 637

Mazeh, T., et al. 2000, ApJ, 532, L55

Moutou, C., Coustenis, A., Schneider, J., Queloz, D., \& Mayor, M. 2003, A\&A, 405, 341

Moutou, C., Coustenis, A., Schneider, J., St. Gilles, R., Mayor, M., Queloz, D. \& Kaufer, A. 2001, A\&A, 371, 260

Narita, N., et al. 2005, PASJ, 57, 471

Richardson, L. J., Deming, D., \& Seager, S. 2003a, ApJ, 597, 581

Richardson, L. J., Deming, D., Wiedemann, G., Goukenleuque, C., Steyert, D., Harrington, J., \& Esposito, L. W. 2003b, ApJ, 584, 1053

Rieke, G. H., et al. 2004, ApJS, 154, 25

Sato, B., et al. 2005, ApJ, 633, 465

Showman, A. P., \& Cooper, C. S. 2006, in Haute Provence Observatory Colloq., Tenth Anniversary of 51-Peg b: Status of and prospects for hot Jupiter studies, ed. L. Arnold, F. Bouchy, \& C. Moutou (Paris: Frontier Group), 242

Showman, A. P., \& Guillot, T. 2002, A\&A, 385, 166

Stern, S. A. 1992, ARA\&A, 30, 185

Vidal-Madjar, A., Lecavelier des Etangs, A., Désert, J.-M., Ballester, G. E., Ferlet, R., Hébrard, G., \& Mayor, M. 2003, Nature, 422, 143

Werner, M. W., et al. 2004, ApJS, 154, 1

Wiedemann, G., Deming, D., \& Bjoraker, G. 2001, ApJ, 546, 1068

Winn, J. N., Suto, Y., Turner, E. L., Narita, N., Frye, B. L., Aoki, W., Sato, B., \& Yamada, T. 2004, PASJ, 56, 655 